\title{
VORTICITY, DIVERGENCE, AND VERTICAL VELOCITY IN A BAROCLINIC BOUNDARY LAYER WITH A LINEAR VARIATION OF THE GEOSTROPHIC WIND
}

\author{
A. WIIN-NIELSEN* \\ The University of Michigan, Michigan, U.S.A.
}

(Received 21 June, 1973)

\begin{abstract}
The Ekman-Taylor problem for the planetary boundary layer is solved in the case of a thermal wind which varies linearly with height. The upper boundary condition is a vanishing ageostrophic wind, while the lower boundary condition is continuity of the stress vector across the interface between the planetary boundary layer and the surface layer. The latter condition is used to determine the magnitude and the direction of the wind at the bottom of the Ekman layer.

Theoretical hodographs are compared with observed hodographs based on five years of observations from Ship $\mathbf{N}$ in the Pacific, giving fair agreement.

The divergence, the vorticity, and the vertical velocity are calculated through the Ekman layer with emphasis on differences between the classical barotropic and the baroclinic cases; these differences are significant, especially in the vertical velocities as compared to the standard approximation.

An extension of the present study to include thermal stratification is desirable.
\end{abstract}

\section{Introduction}

The classical solution for the Ekman layer is normally obtained under a number of simplifying assumptions:

A. The pressure force is constant with height;

B. The kinematic eddy viscosity is constant; and

C. The total acceleration vanishes.

The resulting flow is thus the one which will exist under a balance between the constant pressure force, the Coriolis force and the force of friction.

An important part of the complete solution is the imposed boundary conditions. One of the commonly used conditions is that the a-geostrophic wind vanishes at great height. The boundary condition at the bottom of the Ekman layer is more uncertain. If it is assumed that the Ekman layer reaches all the way to the ground, one may impose the condition that the horizontal velocity vanishes at the ground. Such a condition is often used in classical studies of the Ekman layer, but it is also made in more recent studies (Mahrt and Schwerdtfeger, 1970). Another commonly used boundary condition is based on the existence of a surface (Prandtl) layer in which the stress is constant with height and the vertical shear of the horizontal wind is parallel to the wind itself. The lower boundary condition is then the requirement that the stress vector is continuous at the internal boundary between the Prandtl and the Ekman layers.

This boundary condition is normally divided in two parts: continuity in direction and continuity in magnitude. While the first part of the boundary condition readily

* Present address: European Centre for Medium Range Weather Forecasts, Bracknell, Berks, U.K. 
leads to the determination of one of the integration constants, it is more difficult to satisfy the second part. The reason is that we do not have a good theoretical relation between the magnitude of the horizontal stress and the wind speed under general conditions of thermal stratification in the surface layer. If the lapse rate is adiabatic (neutral stratification), we know that the stress is proportional to the density and the square of the wind speed with the drag coefficient as the proportionality factor. Such a relation is also assumed if the stratification is non-neutral, but it must then be kept in mind that the relation is of an empirical nature.

In view of these difficulties, it is often decided (Taylor, 1915, 1916) to let the remaining integration constant, normally expressed as the angle $\alpha_{0}$ between the wind at the bottom of the Ekman layer and the geostrophic wind, remain undetermined. However, if we adopt the empirical relation between the stress and the wind, it is relatively straightforward to calculate $\alpha_{0}$. Values of $\alpha_{0}$ around $20^{\circ}$ are obtained for reasonable values of the drag coefficient and the kinematic eddy viscosity.

Numerous investigations of the Ekman flow have been made under somewhat more general conditions than those stated above. Godske et al. (1957), give an excellent survey of such investigations. It is, for example, relatively easy to replace condition A which says that the geostrophic wind is constant with height by a linear variation with height of the geostrophic wind. Keeping assumptions $\mathrm{B}$ and $\mathrm{C}$, one obtains readily the formal solution, but it becomes cumbersome to satisfy the lower boundary condition if we require continuity in the stress vector. The angle $\alpha_{0}$ will now depend on the magnitude and the direction of the thermal geostrophic wind.

The purpose of the present paper is to present the solution to the problem just stated. The mathematical solution is certainly neither new (see MacKay (1971) in addition to other papers) nor difficult, but we shall put special emphasis on the variation of $\alpha_{0}$ with regard to the parameters of the problem and on the vertical velocity, divergence, and vorticity in the boundary layer, where the baroclinicity has been incorporated through a simple linear variation of the geostrophic wind with altitude. It is realized that while the qualitative analysis of the baroclinic effects appears correct, the quantitative results depend heavily on the simple model.

\section{The Formal Solution}

The geostrophic wind will be assumed to vary according to the formula

$$
\mathbf{v}_{g}=\mathbf{v}_{g 0}+\mathbf{v}_{T} z
$$

where $\mathbf{v}_{g 0}$ is the geostrophic wind at the lower boundary, $\mathbf{v}_{T}$ the geostrophic thermal wind, and $z$ is height.

The equations for the Ekman flow are

$$
\begin{aligned}
& \frac{\partial^{2} u}{\partial z^{2}}+\frac{f}{K}\left(v-v_{g 0}-v_{T} z\right)=0 \\
& \frac{\partial^{2} v}{\partial z^{2}}-\frac{f}{K}\left(u-u_{g 0}-u_{T} z\right)=0
\end{aligned}
$$


in which $f$ is the Coriolis parameter, $K$ the (constant) kinematic eddy viscosity, and $u$ and $v$ the horizontal wind components. The formal solution of (2.2) is straightforward. Using the upper boundary condition that the wind should approach the geostrophic wind at great heights, we may write the solution in the form

$$
\begin{aligned}
u & =u_{g 0}+H u_{T} \eta+e^{-\eta}\left[\left(u_{0}-u_{g 0}\right) \cos \eta+\left(v_{0}-v_{g 0}\right) \sin \eta\right] \\
v & =v_{g 0}+H v_{T} \eta+e^{-\eta}\left[\left(v_{0}-v_{g 0}\right) \cos \eta-\left(u_{0}-u_{g 0}\right) \sin \eta\right]
\end{aligned}
$$

where $\left(u_{0}, v_{0}\right)$ is the wind at $z=0, H=(2 K / f)^{1 / 2}$, and $\eta=z / H$.

\section{The Boundary Conditions}

The boundary condition requires continuity in the horizontal stress vector across the boundary between the Prandtl layer and the Ekman layer, i.e., at $\eta=0$. Since no turning of the wind takes place in the Prandtl layer, we find as usual at $\eta=0$ that

$$
\frac{\partial u}{\partial \eta}=\frac{\partial v}{v \eta}, \quad \eta=0 .
$$

Using (2.3) we find from (3.1) that

$$
V_{0}=V_{g 0}\left(\cos \alpha_{0}-\sin \alpha_{0}\right)+H V_{T} \sin \left(\alpha_{T}-\alpha_{0}\right)
$$

where the various quantities are defined through the relations

$$
\begin{array}{ll}
u_{0}=V_{0} \cos \alpha_{0}, & v_{0}=V_{0} \sin \alpha_{0}, \\
u_{T}=V_{T} \cos \alpha_{T}, & v_{T}=V_{T} \sin \alpha_{T} .
\end{array}
$$

(See Figure 1.)

The remaining integration constant is $\alpha_{0}$. It is determined by making use of the relation

$$
\tau=\varrho K\left|\frac{\partial v}{\partial z}\right|=\varrho c_{d} V_{0}^{2}, \quad \eta=0
$$

in which $c_{d}$ is the drag coefficient.

Making use of (2.3), we find after tedious calculations:

$$
\begin{aligned}
G\left(\alpha_{0}\right)=B\left[\cos \alpha_{0}-\sin \alpha_{0}-A \sin \left(\alpha_{0}-\alpha_{T}\right)\right]^{2}+ \\
\quad-A\left[\cos \left(\alpha_{0}-\alpha_{T}\right)+\sin \left(\alpha_{0}-\alpha_{T}\right)\right]-2 \sin \alpha_{0}=0
\end{aligned}
$$

where

$$
A=\frac{V_{T}}{V_{s 0}}\left(\frac{2 K}{f}\right)^{1 / 2}
$$

and

$$
B=\sqrt{2} c_{d} \frac{V_{g 0}}{(K f)^{1 / 2}} .
$$




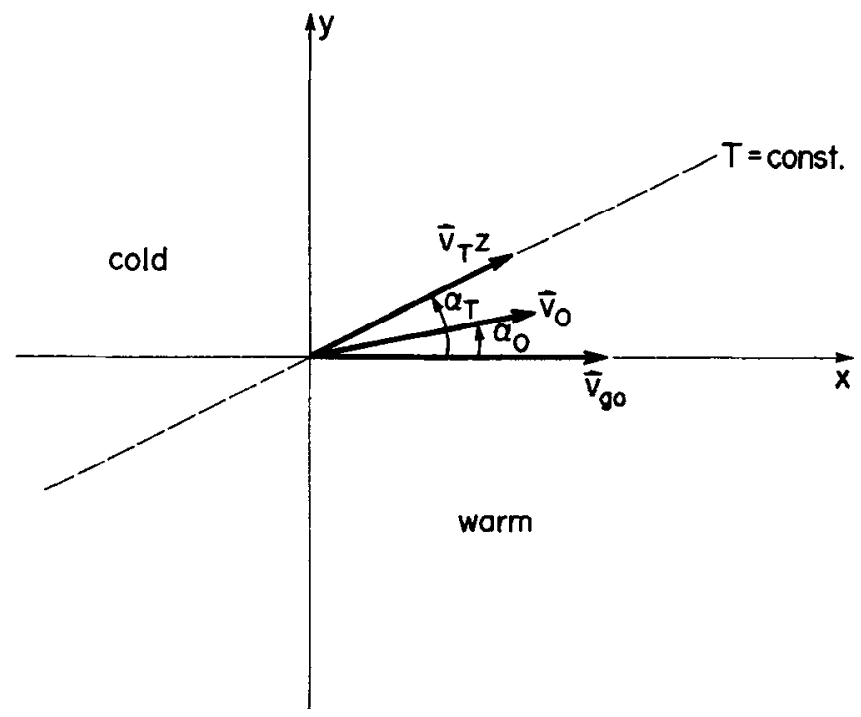

Fig. 1. Explanation of notations. $\mathrm{V}_{g 0}$ is along the abscissa; $\mathrm{V}_{T} \cdot Z$ along the isotherm forming an angle $\alpha_{T}$ with the $x$-axis. $V_{0}$ is the surface wind forming an angle $\alpha_{0}$ with $V_{g 0} . \alpha_{T}>0$ occurs for cold air advection, $\alpha_{T}<0$, for warm air advection.

If $V_{T}=\alpha_{T}=0$, we find the classical case

$$
F\left(\alpha_{0}\right)=\frac{\sin \alpha_{0}}{1-\sin \left(2 \alpha_{0}\right)}
$$

which is simple to solve by graphical procedures.

The numerical values used in the calculations to be reported in the following sections are: $V_{g 0}=10 \mathrm{~s}^{-1}, K=5 \mathrm{~m}^{2} \mathrm{~s}^{-1}, f=10^{-4} \mathrm{~s}^{-1}, c_{d}=2.5 \times 10^{-3}$, while $V_{T}$ and $\alpha_{T}$ were varied. We find:

$$
B=1.52, \quad A=31.62 V_{T}
$$

where $V_{T}$ is measured in $\mathrm{s}^{-1}$.

\section{Determination of $\alpha_{0}$}

The graphical solution of (3.8) is illustrated in Figure 2 which shows $F\left(\alpha_{0}\right)$ as a function of $\alpha_{0}$. Corresponding to $B=1.52$, we find that $\alpha_{0}=18^{\circ}$ which therefore is the typical angle between the surface wind and the geostropic wind for the selected numerical values of the parameters. It is seen from (3.7), (3.8), and Figure 2 that $\alpha_{0}$ increases with $c_{d}$ and $V_{g 0}$, but decreases with $K$ and $f$. These relations are well-known and have been discussed by other authors, see, for example, Petterssen (1956) and Haltiner and Martin (1957), who show comparisons between computed and observed values of $\alpha_{0}$ although none of the authors demonstrates in detail how $\alpha_{0}$ is computed.

We shall next turn our attention to (3.5). Equation (3.5) was solved by transforming it into a fourth-degree equation because a standard computer program exists for the 


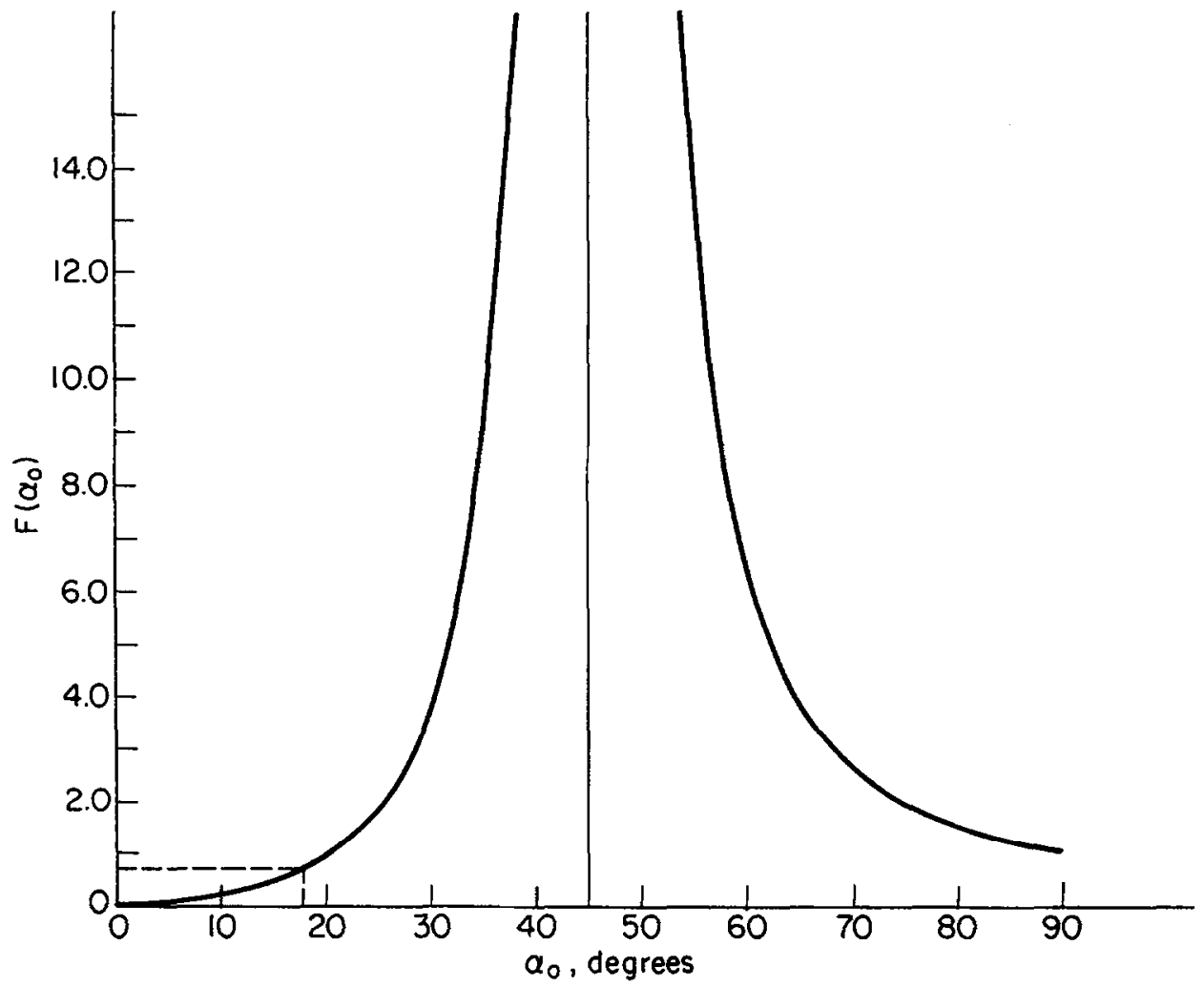

Fig. 2. $F\left(\alpha_{0}\right)$, given in (3.8), as a function of $\alpha_{0}$.

solution of such an equation. Introducing

$$
\xi=\tan \alpha_{0}
$$

we may write (3.5) in the form:

$$
\begin{aligned}
\left(\beta^{2}-\varepsilon^{2}\right) \xi^{4}-2(\beta \sigma+\delta \varepsilon) \xi^{3}+ & \left(\sigma^{2}+2 \alpha \beta-\delta^{2}-\varepsilon^{2}\right) \xi^{2}+ \\
& -2(\alpha \sigma+\delta \varepsilon) \xi+\left(\alpha^{2}-\delta^{2}\right)=0
\end{aligned}
$$

where

$$
\begin{aligned}
& \alpha=B\left(1+A \sin \alpha_{T}\right)^{2} \\
& \beta=B\left(1+A \cos \alpha_{T}\right)^{2} \\
& \sigma=2 B\left(1+A \sin \alpha_{T}\right)\left(1+A \cos \alpha_{T}\right) \\
& \delta=A\left(\cos \alpha_{T}-\sin \alpha_{T}\right) \\
& \varepsilon=2+A\left(\cos \alpha_{T}+\sin \alpha_{T}\right) .
\end{aligned}
$$

Equation (4.2) was solved by numerical methods for given values of $B, A$, and $\alpha_{T}$. Among the four roots of (4.2), we selected the two real roots which existed in all cases. One of these seems to be a spurious root because it has large negative values corresponding to a cross-isobaric flow from low to high pressure. The calculations 
were done by selecting the standard values of $B$ quoted just after (3.8). $A$ had the values calculated by setting $V_{T}=4 \times 10^{-3} \mathrm{~s}^{-1}$. For these values, we let $\alpha_{T}$ vary from $-180^{\circ}$ to $+180^{\circ}$ in increments of $10^{\circ}$. The resulting values of $\alpha_{0}$ are shown in Figure 3. It is seen that $\alpha_{0}$ varies around the standard value corresponding to $V_{T}=0$ and $\alpha_{T}=0$ as found in Figure 2. Warm-air advection $\left(\alpha_{T}<0\right)$ gives relatively small values of $\alpha_{0}$, while cold-air advection $\left(\alpha_{T}>0\right)$ gives the largest values of $\alpha_{0}$.

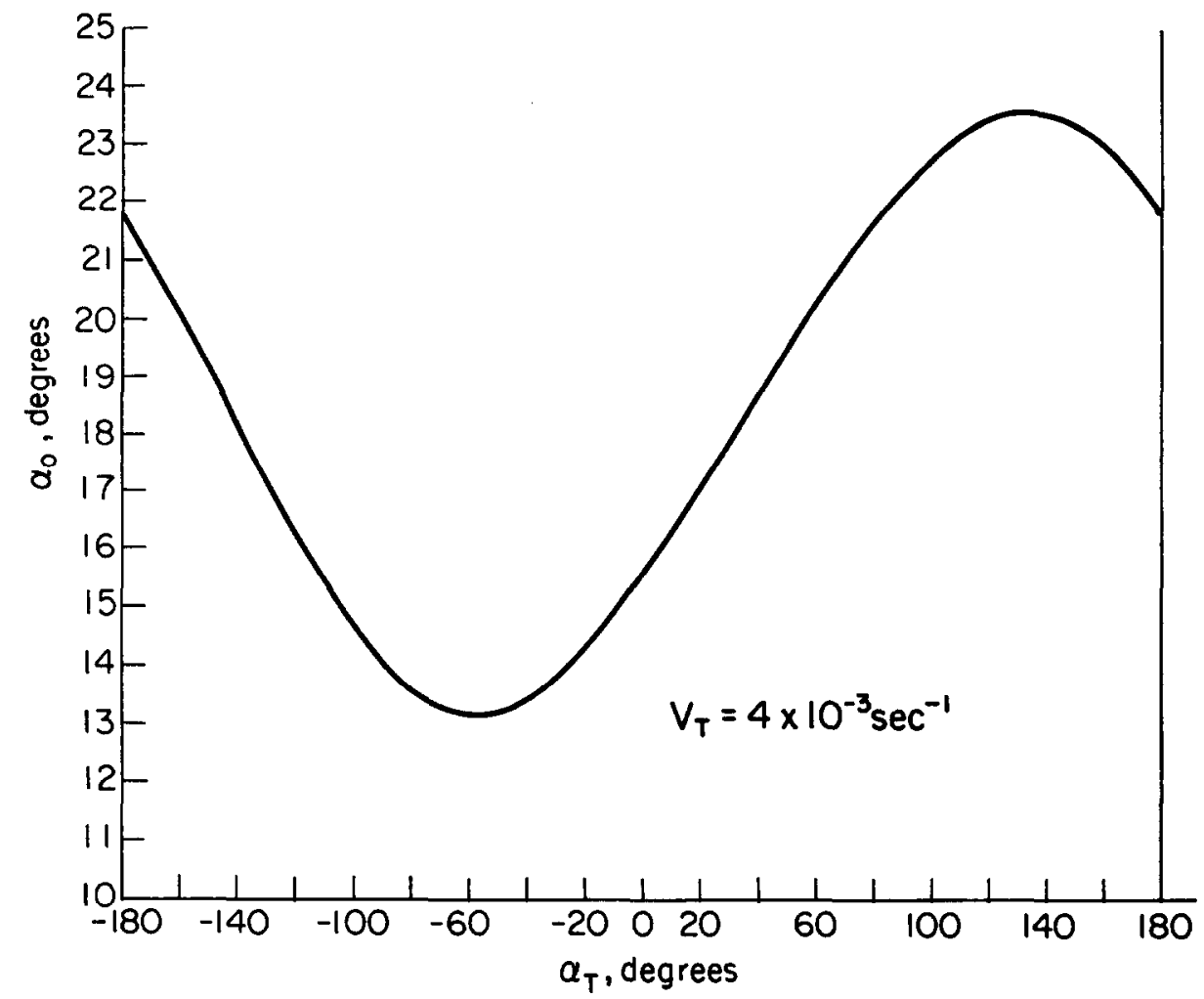

Fig. 3. $\alpha_{0}$ as a function of $\alpha_{T}$ for $V_{g 0}=10 \mathrm{~m} \mathrm{~s}^{-1}$ and $V_{T}=4 \times 10^{-3} \mathrm{~s}^{-1}$ obtained as a solution of (3.5).

\section{The Hodographs}

The solutions obtained in Sections 3 and 4 show that the wind vector $v$ at the upper limit of the planetary boundary layer will approach the wind field $v_{g 0}+v_{T} Z$, which is the geostrophic field in the model. A number of hodographs have been computed as examples.

Figure 4 shows the hodograph for a case where $\alpha_{T}=0$ and $V_{T}=4 \times 10^{-3} \mathrm{~s}^{-1}$. In all the hodograph calculations, we have used the values $f=10^{-4} \mathrm{~s}^{-1}, V_{g 0}=10 \mathrm{~m} \mathrm{~s}^{-1}$, and $K=5 \mathrm{~m}^{2} \mathrm{~s}^{-1}$. This case corresponds to an increase of wind with height without change of direction. The hodograph of the geostrophic wind is the $x$-axis. It is seen that the hodograph of the wind approaches the hodograph of the geostrophic wind, and that the two hodographs are extremely close to each other when $\eta$ has a value of approxim- 


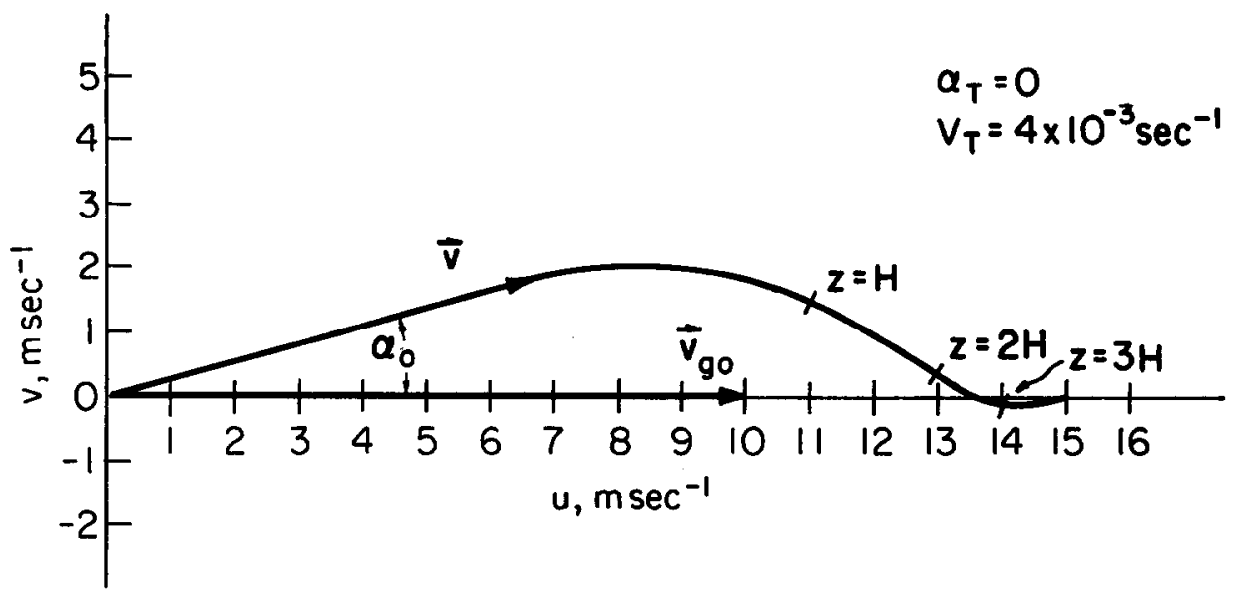

Fig. 4. Computed hodograph for $\alpha_{T}=0, V_{T}=4 \times 10^{-3} \mathrm{~s}^{-1}$, and $V_{g 0}=10 \mathrm{~m} \mathrm{~s}^{-1}$.

ately 3 or $Z=3 H$. It has been customary to use $Z=\pi H$ as the effective depth of the planetary boundary layer. This selection of the height beyond which most of the influence of the lower boundary has vanished is justified also in our case, including a thermal wind.

Figure 5, shows the hodographs for $\alpha_{T}= \pm 40^{\circ}$ and $V_{T}=4 \times 10^{-3} \mathrm{~s}^{-1}$. The geostrophic hodographs are straight lines forming an angle of $\pm 40^{\circ}$ with the abscissa. The hodograph of the wind approaches the geostrophic hodograph, and the two are practically identical for $\eta=3-4$. Figure 6 shows the geostrophic and actual hodographs for the case $\alpha_{T}= \pm 90^{\circ}$ and $V_{T}=4 \times 10^{-3} \mathrm{~s}^{-1}$. We notice here as in Figure 5 that the wind becomes slightly larger than the geostrophic wind for values of $\eta$ larger than about 2 .

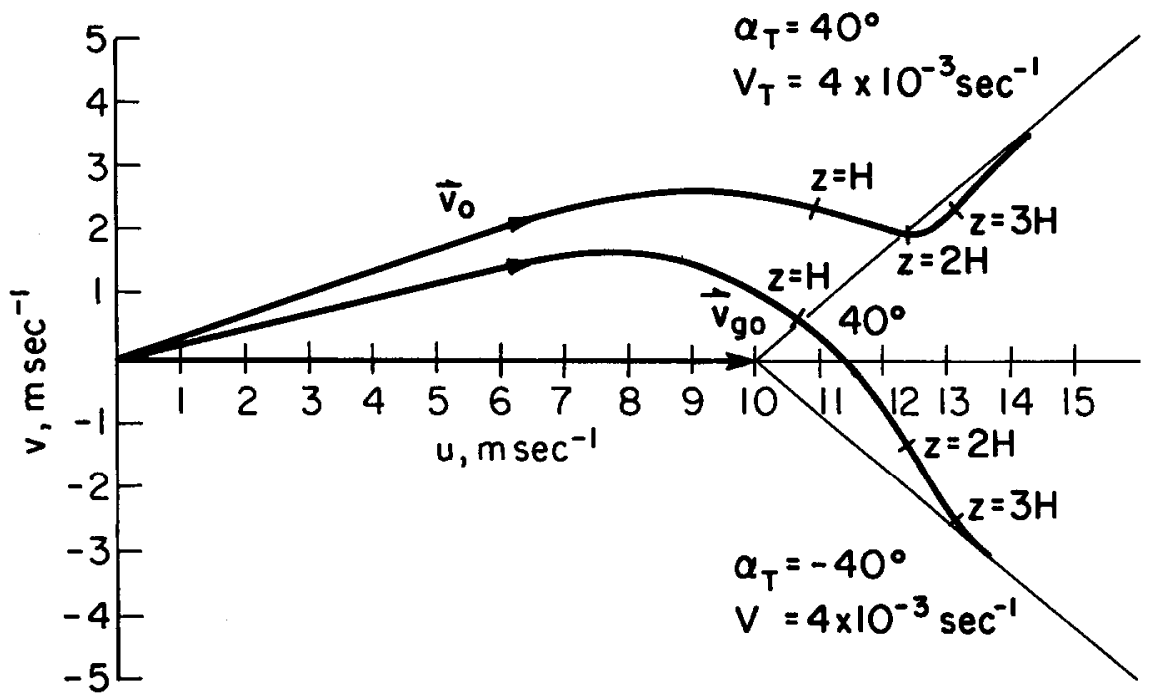

Fig. 5. Computed hodographs for $\alpha_{T}= \pm 40^{\circ}, V_{T}=4 \times 10^{-3} \mathrm{~s}^{-1}$, and $V_{g 0}=10 \mathrm{~m} \mathrm{~s}^{-1}$. 


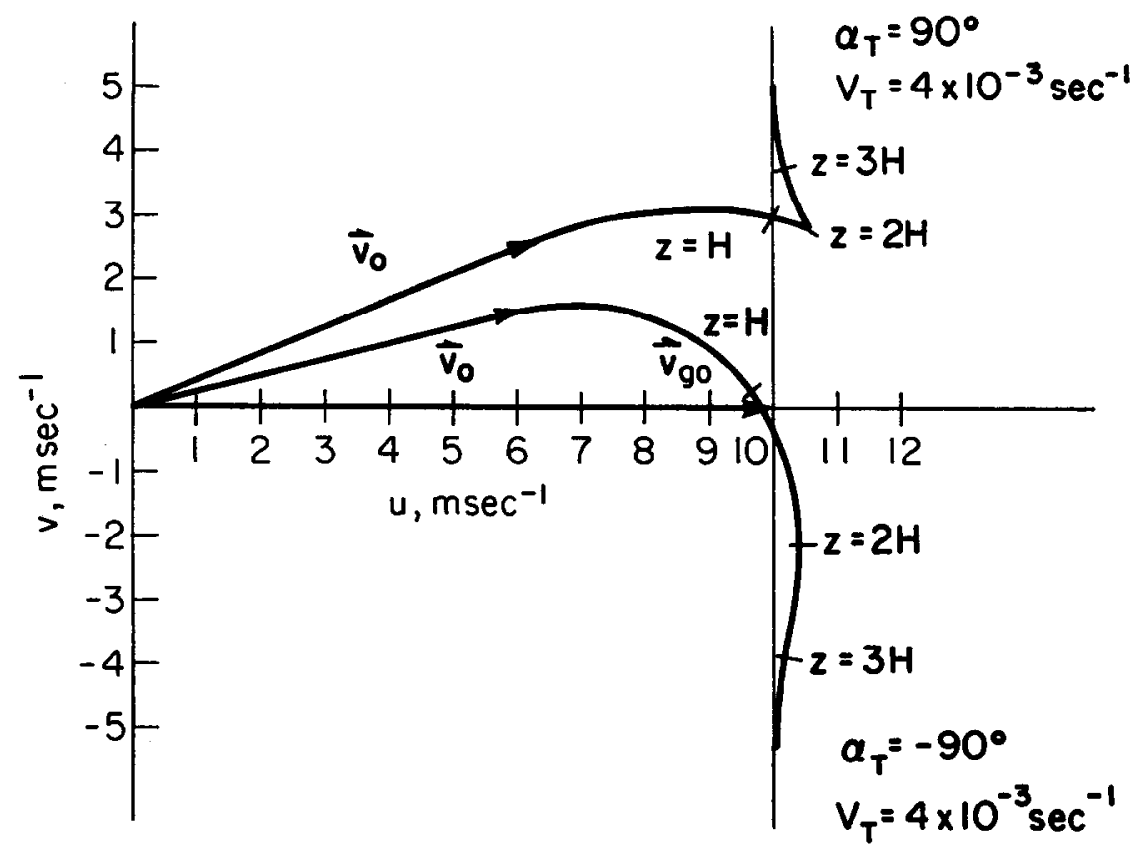

Fig. 6. Computed hodographs for $\alpha_{T}= \pm 90^{\circ}, V_{T}=4 \times 10^{-3} \mathrm{~s}^{-1}$, and $V_{g 0}=10 \mathrm{~m} \mathrm{~s}^{-1}$

Figures 4-6 show clearly the main effect of the thermal wind on the Ekman-layer solution. It is obvious that there are significant differences between cold and warm air advection. If the thermal wind has been incorporated in an essentially correct manner, it should be possible to verify the modifications of the thermal wind by a comparison with atmospheric data. Mendenhall (1967) has analyzed data for a period of five years for various stations, including data from some of the permanent ocean weather ships. He has made a particularly detailed analysis for the five-year period, 1960-64, for ship $\mathrm{N}$, located at approximately $140^{\circ} \mathrm{W}$ and $30^{\circ} \mathrm{N}$. The observed hodographs for each $30^{\circ}$ in surface wind direction are reproduced in Figure 7, which contains the number of cases in each category and the veering (positive for clockwise turning) of the observed wind in the layer from the surface to $1 \mathrm{~km}$. The elevation marks on each hodograph denote the heights 0 (surface), $150,300,500,1000,1500$, and $2000 \mathrm{~m}$. It is obvious that the prevailing wind direction is from northeast and east-northeast because these two directions contain 2340 of the total 5358 cases which go into the statistics. It is stated that 'the climatological horizontal temperature gradient at ship $\mathrm{N}$ varies little throughout the year and is directed with warmest temperatures toward the south.' This means in our formulation that the thermal wind is directed from west to east.

We may now adapt our theoretical solution to this case. We note that the solution applies in a coordinate system in which the surface geostrophic wind points along the abscissa. In order to obtain the solution in the ordinary coordinate system where the abscissa points toward east and the ordinate toward north, we must therefore turn the 
original coordinate system through an angle $-\alpha_{T}$ which is determined in the following way: we know the directions $\alpha_{0}^{*}$ for the hodographs in Figure 7, i.e., $\alpha_{0}^{*}=75^{\circ}, 45^{\circ}$, $15^{\circ}, \ldots, 105^{\circ}$. It is also seen that $\alpha_{0}^{*}=\alpha_{0}-\alpha_{T}$. The relation between $\alpha_{0}$ and $\alpha_{T}$ is given in Figure 3. The quantity $\alpha_{0}-\alpha_{T}$ computed from Figure 3 is a monotonic function of $\alpha_{T}$ which therefore can be found by a simple graphical procedure knowing $\alpha_{0}^{*}$. Figure 3

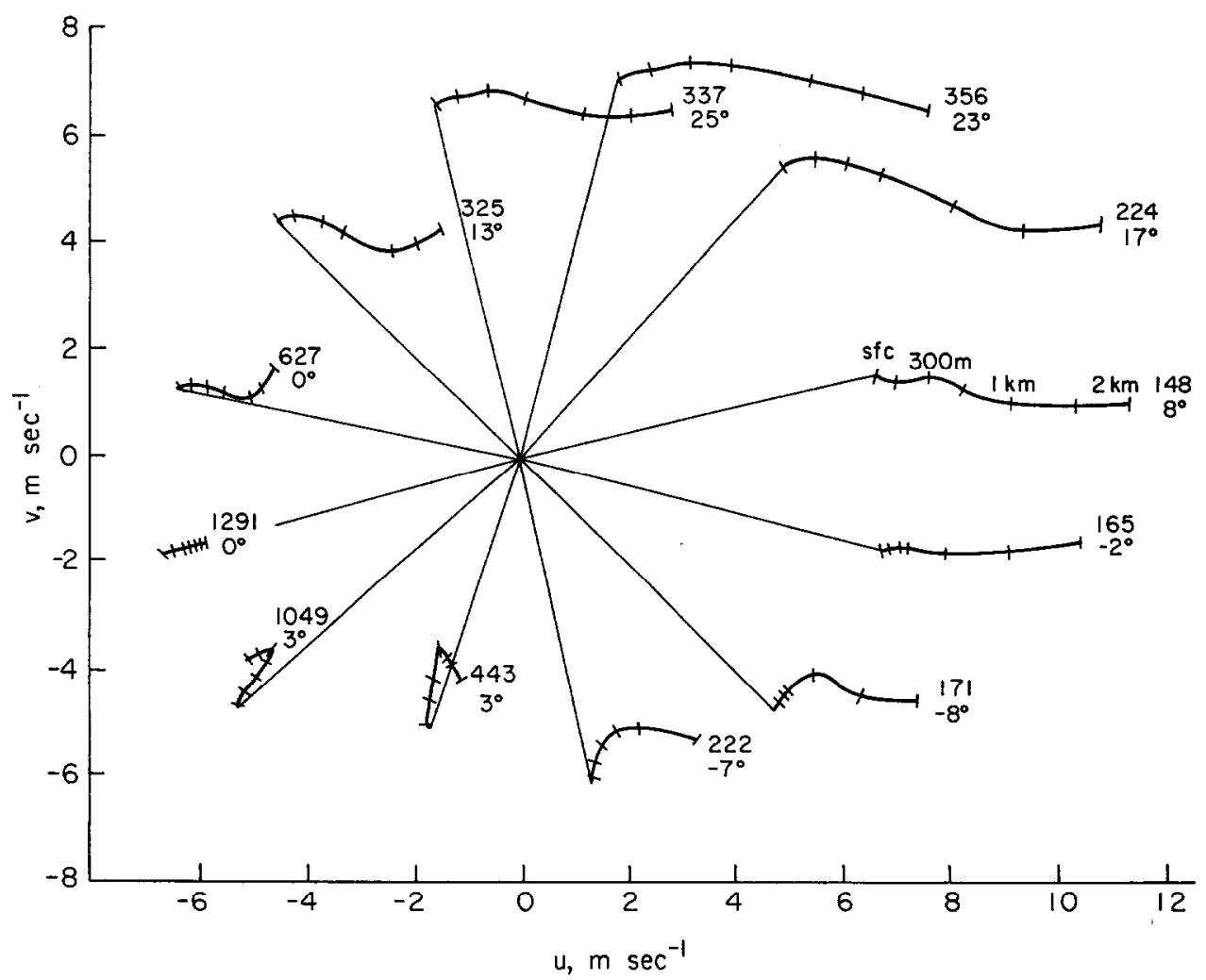

Fig. 7. Observed hodographs at ship $\mathrm{N}\left(140^{\circ} \mathrm{W}, 30^{\circ} \mathrm{N}\right)$ for the years $1960-64.12$ hodographs are shown, grouped according to surface wind direction. Upper number shows the number of observations, while the lower number gives the observed veering between the surface and $1 \mathrm{~km}$, counted positive in a clockwise direction. Elevation marks are shown at 0 (surface), 150, 300, 500, 1000, 1500, and $2000 \mathrm{~m}$ (after Mendenhall, 1967).

then gives $\alpha_{0}$. The derived values of $\alpha_{T}$ and $\alpha_{0}$ are used to calculate $u$ and $v$. These values are finally converted to the ordinary system using the expressions:

$$
\begin{aligned}
& u_{*}=u \cos \alpha_{T}+v \sin \alpha_{T} \\
& v_{*}=-u \sin \alpha_{T}+v \cos \alpha_{T} .
\end{aligned}
$$

Using $V_{g 0}=10 \mathrm{~m} \mathrm{~s}^{-1}, V_{T}=4 \times 10^{-3} \mathrm{~s}^{-1}, f=0.73 \times 10^{-4} \mathrm{~s}^{-1}$ and $K-3.6 \mathrm{~m}^{2} \mathrm{~s}^{-1}$ (giving the same value of $H$ as before), we have computed the theoretical hodographs shown in Figure 8. Considering the lack of precise information on $V_{g 0}$ and $V_{T}$, it is seen that there is good qualitative agreement between the theoretical and observed hodo- 


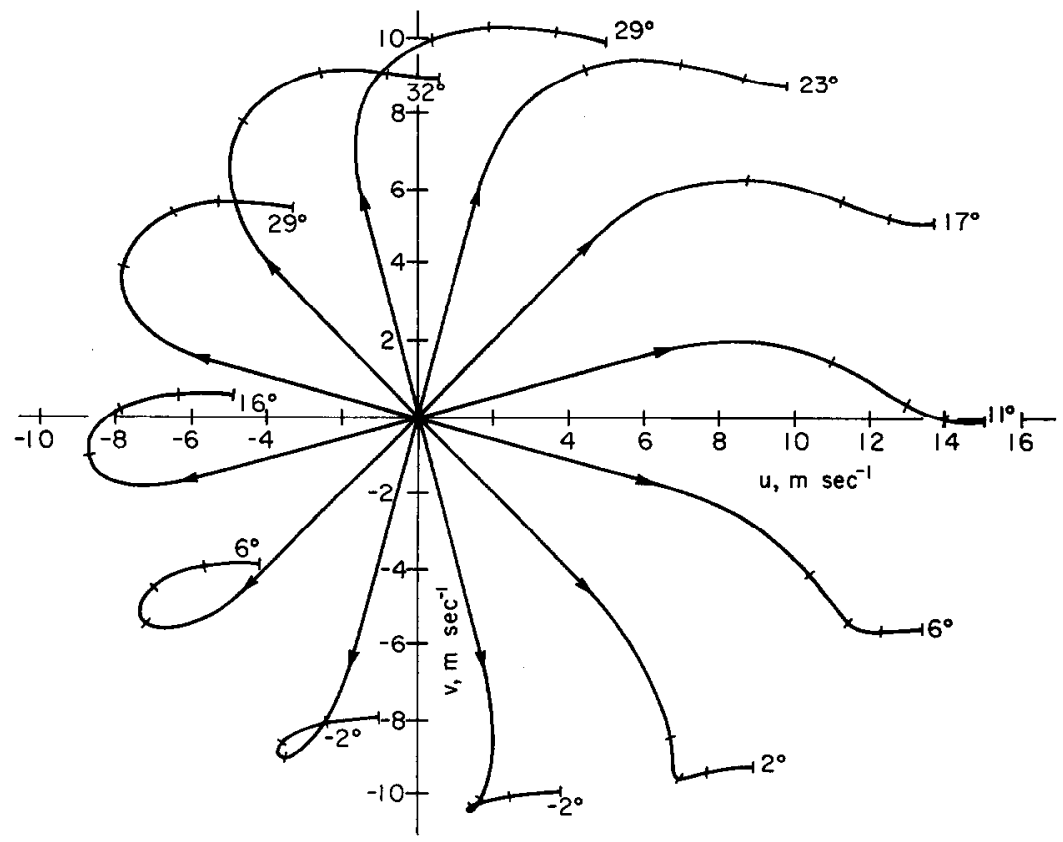

Fig. 8. Theoretical hodographs computed with $V_{g 0}=10 \mathrm{~m} \mathrm{~s}^{-1}, V_{T}=4 \times 10^{-3} \mathrm{~s}^{-1}, f=0.729 \times 10^{-4} \mathrm{~s}^{-1}$ and $K=3.6 \mathrm{~m}^{2} \mathrm{~s}^{-1}$. Elevation marks are shown at $\eta=0,1,2,3$, and 4 . Arrangement as in Figure 7 . The veering is calculated for the layer from 150 to $1000 \mathrm{~m}$.

graphs, providing a reasonable verification of the modifications to the classical Ekman-Taylor spiral caused by the thermal wind. Note, in particular, the much improved verification as compared to the theoretical hodographs computed by Mendenhall (1967) who uses the same basic solution as presented here, but with the boundary condition $u_{0}=v_{0}=0$.

It is shown in Mendenhall's (1967) statistical analysis that the thermal wind and the lapse rate are the major factors responsible for modifications in the classical EkmanTaylor spirals. Of these we have incorporated the first factor only. The second factor is particularly important over land with much larger diurnal changes in lapse rate than over sea. However, it is evident that the static stability must be incorporated in the analysis in order to account for the observed changes of the wind.

\section{Divergence, Vorticity and Vertical Velocity}

The cross-isobaric flow implied by all the hodographs indicates as usual that we have a mass convergence in the low-pressure areas and a divergence in the high-pressure areas. It follows that there exists an induced vertical velocity at the top of the planetary boundary layer created by the stress within the layer. This process has for a long time (Charney and Eliassen, 1949) been used to incorporate the influence of the planetary boundary layer into the models of the large-scale flow of the atmosphere. 
It is therefore of interest to investigate any additional effect created by the thermal wind which has been included in this study.

The most straightforward way to do this is to start from the solution for $u$ and $v$. However, in order to work conveniently with these equations, it is advantageous to write them as a single vector equation. We use for this purpose the coordinate system in which the abscissa is along the geostrophic wind direction and the ordinate is pointing in the direction of the vector $\mathbf{k} \times \mathbf{v}_{g 0}$. We get:

$$
\begin{aligned}
\mathbf{v}=\mathbf{v}_{g 0} & +H_{\eta} \mathbf{v}_{T}+\sqrt{2} \sin \alpha_{0} \cos \left(\alpha_{0}+\frac{3 \pi}{4}-\eta\right) e^{-\eta} \mathbf{v}_{g 0} \\
& +\sqrt{2} \sin \alpha_{0} \sin \left(\alpha_{0}+\frac{3 \pi}{4}-\eta\right) e^{-\eta} \mathbf{k} \times \mathbf{v}_{g 0} \\
& +H \frac{V_{T}}{V_{g 0}} \sin \left(\alpha_{T}-\alpha_{0}\right) \cos \left(\alpha_{0}-\eta\right) e^{-\eta} \mathbf{v}_{g 0} \\
& +H \frac{V_{T}}{V_{g 0}} \sin \left(\alpha_{T}-\alpha_{0}\right) \sin \left(\alpha_{0}-\eta\right) e^{-\eta} \mathbf{k} \times \mathbf{v}_{g 0} .
\end{aligned}
$$

We shall first derive the divergence of $\mathbf{v}$. In so doing, we note that the geostrophic wind has no divergence under our simplifying assumption that the Coriolis parameter may be considered as constant. The same holds for the geostrophic thermal wind. Furthermore, we remark that

$$
\nabla \cdot\left(\mathbf{k} \times \mathbf{v}_{g 0}\right)=-\mathbf{k} \cdot \nabla \times \mathbf{v}_{g 0}=-\zeta_{g 0}
$$

where $\zeta_{g 0}$ is the vorticity of the surface geostrophic wind. We assume finally for simplicity that the horizontal variations in the wind speeds, $V_{g 0}$ and $V_{T}$ as well as in the direction $\alpha_{T}$ of $\mathbf{v}_{T}$ relative to $\mathbf{v}_{g 0}$, are small compared to variation in the direction of $\mathbf{v}_{g 0}$. These conditions are certainly satisfied if the isobars and isotherms are concentric circles, in which case $\alpha_{T}=0$ and $V_{g 0}$ and $V_{T}$ are assumed to be constants, but they will also hold approximately under more general conditions. After these preparations we get:

$$
\begin{aligned}
\frac{\nabla \cdot \mathbf{v}}{\zeta_{g}}= & -\sqrt{2} \sin \alpha_{0} \sin \left(\alpha_{0}+\frac{3 \pi}{4}-\eta\right) e^{-\eta}+ \\
& -H \frac{V_{T}}{V_{g 0}} \sin \left(\alpha_{T}-\alpha_{0}\right) \sin \left(\alpha_{0}-\eta\right) e^{-\eta}
\end{aligned}
$$

We shall next derive an expression for the vorticity $\zeta=\mathbf{k} \cdot(\nabla \times \mathbf{v})$ of the flow. In this connection we note that

$$
\mathbf{k} \cdot\left[\nabla \times\left(\mathbf{k} \times \mathbf{v}_{g 0}\right)\right]=\nabla \cdot \mathbf{v}_{g 0}=0
$$

and we get:

$$
\begin{aligned}
\frac{\zeta}{\zeta_{g 0}}=1 & +H \frac{\zeta_{T}}{\zeta_{g 0}} \eta+\sqrt{2} \sin \alpha_{0} \cos \left(\alpha_{0}+\frac{3 \pi}{4}-\eta\right) e^{-\eta}+ \\
& +H \frac{V_{T}}{V_{g 0}} \sin \left(\alpha_{T}-\alpha_{0}\right) \cos \left(\alpha_{0}-\eta\right) e^{-\eta}
\end{aligned}
$$


We may finally obtain an expression for the vertical velocity in the boundary layer. This expression is obtained from the continuity equation which in our simple formulation is

$$
\frac{\partial w}{\partial z}=-\nabla \cdot \mathbf{v}
$$

Equation (6.3) is introduced on the right-hand side of (6.6) and the resulting equation is integrated with respect to height using the boundary condition that $w=0$ at $z=0$. We get after elementary integrations:

$$
\begin{aligned}
\frac{w}{H \zeta_{g}}= & \sin \alpha_{0} \cos \alpha_{0}-\frac{H V_{T}}{\sqrt{2} V_{g 0}} \sin \left(\alpha_{T}-\alpha_{0}\right) \cos \left(\frac{\pi}{4}+\alpha_{0}\right)-\sin \alpha_{0} \times \\
& \times \cos \left(\alpha_{0}-\eta\right) e^{-\eta}+\frac{H V_{T}}{\sqrt{2} V_{g 0}} \sin \left(\alpha_{T}-\alpha_{0}\right) \cos \left(\frac{\pi}{4}+\alpha_{0}-\eta\right) e^{-\eta}
\end{aligned}
$$

As is normally the case, we may for practical purposes neglect the terms containing the exponential function for sufficiently large values of $\eta$. We find therefore that the vertical velocity at the top of the Ekman layer is, approximately:

$$
\frac{w_{E}}{H \zeta_{g}}=\sin \alpha_{0} \cos \alpha_{0}-\frac{H V_{T}}{\sqrt{2} V_{g 0}} \sin \left(\alpha_{T}-\alpha_{0}\right) \cos \left(\frac{\pi}{4}+\alpha_{0}\right) .
$$

The first term is identical to the vertical velocity found at the top of the Ekman layer without a thermal wind. The second term is due to the assumed baroclinicity of the boundary layer.

Equations (6.3), (6.5), and (6.7) can be used to investigate the distribution of divergence, vorticity, and vertical velocity through the depth of the baroclinic Ekman boundary layer. Such a distribution will be shown and discussed at the end of this section. We shall first consider (6.8) which gives the vertical velocity at the top of the Ekman boundary layer. Figure 9 shows the relative variation of $w_{E}$, computed from (6.8), as a percentage of a standard value $w_{S}$, computed from the formula

$$
w_{s}=H \zeta_{g} \sin \alpha_{0} \cos \alpha_{0}
$$

in which we have assumed $\alpha_{0}=18^{\circ}$ as obtained in Figure 2. As seen from the figure, we get values of $w_{E}$ as low as $60 \%$ of the standard value and as high as $130 \%$ when $\alpha_{T}$ is large and positive. It is intuitively clear that the results must turn out this way because a negative value of $\alpha_{T}$ creates a reduced cross-isobaric mass flux as seen from Figures 5 and 6, while a positive value of $\alpha_{T}$ enhances the mass flux.

We shall finally present the vertical distributions of divergence, vorticity and vertical velocity in the Ekman layer. They were computed from (6.3), (6.5), and (6.7), repectively, with $V_{T}=4 \times 10^{-3} \mathrm{~s}^{-1}$ and $\alpha_{T}=-90^{\circ}$. Several other values of $V_{T}$ and $\alpha_{T}$ were also used, but the major aspects of the results are the same because the thermal wind 
incorporated in this study is a correction factor. Figure 10 shows the vertical distribution of $\nabla \cdot v / \zeta_{g 0}$ as a function of $\eta$. The solid curve is from (6.3), while the dashed curve is obtained from the first term in the equation (the barotropic boundary layer). The largest values of the convergence are found in the lowest layer with a maximum at

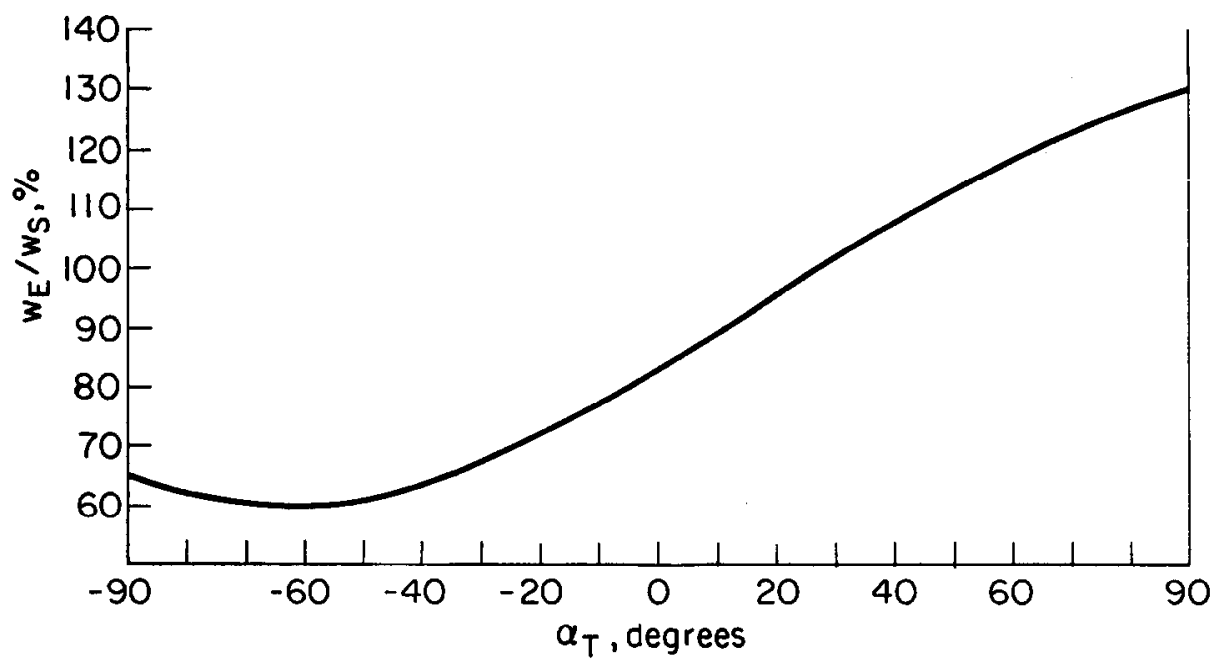

Fig. 9. The ratio of the vertical velocity $w_{E}$ at the top of the planetary boundary layer and the standard vertical velocity ws, computed from the barotropic layer, as a function of $\alpha_{T}$.

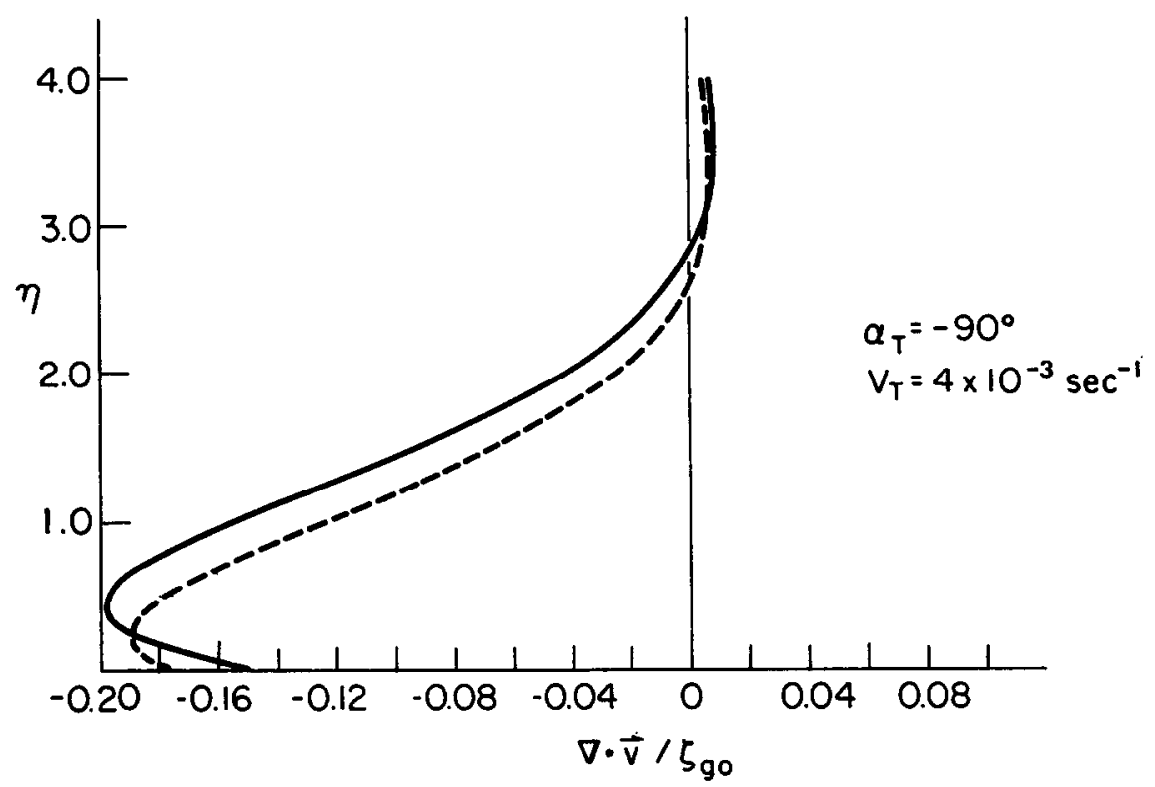

Fig. 10. The divergence of the Ekman solution as a function of height computed from (6.3). Dashed line represents the barotropic solution. 
$\eta=0.5$ (approximately $150 \mathrm{~m}$ ) with rapidly decreasing values for larger values of $\eta$.

Figure 11 shows the vorticity of the Ekman flow as a function of $\eta$ for the same case as in Figure 10. The solid curve depicts the variation in the baroclinic Ekman layer in which the vorticity approaches the sloping straight line which represents the vorticity of the baroclinic, geostrophic flow. The dashed curve is the vorticity which exists in the barotropic Ekman layer for which the asymptotic value is the vorticity of the constant geostrophic wind.

Figure 12 shows in a similar way the vertical profile of the vertical velocity in the baroclinic and barotropic cases, respectively. Both of these curves approach an asymptotic value as $\eta$ becomes large, but the barotropic curve has a smaller asymp-

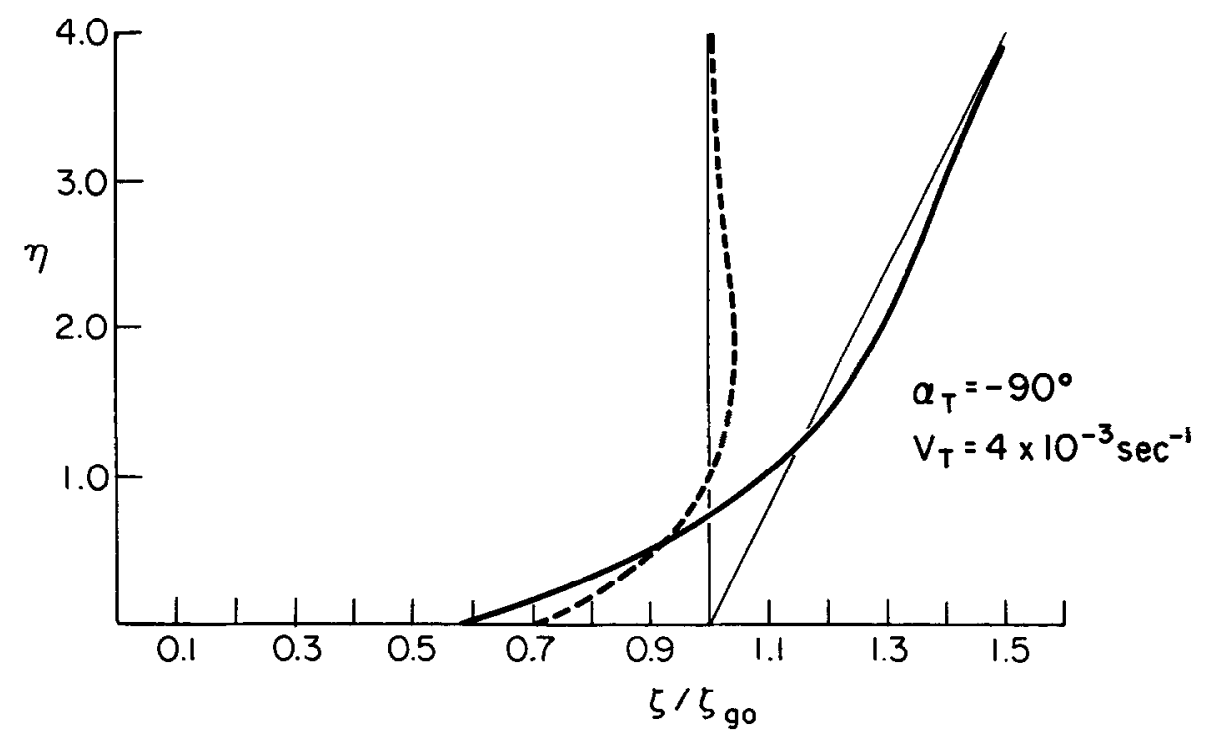

Fig. 11. The vorticity of the Ekman solution as a function of height computed from (6.5). Dashed line represents the barotropic solution.

totic value than the baroclinic curve. Note, that there is no discrepancy between Figures 12 and 9 because the dashed curve is computed for the same value, $\alpha_{0} \approx 14^{\circ}$, as for the baroclinic curve while the standard of comparison in Figure 9 is $\alpha_{0}=18^{\circ}$.

In Figure 13 we show, for the sake of completeness, the ratio $-\nabla \cdot \mathbf{v} / \zeta$ as a function of $\eta$. As expected, the largest values are found in the lowest layers where the ratio becomes as large as 0.25 .

It is furthermore of interest to calculate the divergence and the vorticity at $\eta=0$ as a function of $\alpha_{T}$. Figure 14 displays the ratio $-(\nabla \cdot v) / \zeta_{g 0}$ at $\eta=0$. The figure shows that the ratio is smallest for $\alpha_{T}=-70^{\circ}$, but increases to 0.25 for $\alpha_{T}=90^{\circ}$. The dashed curve in Figure 14 shows the variation of the ratio if we include only the first term in (6.3). 


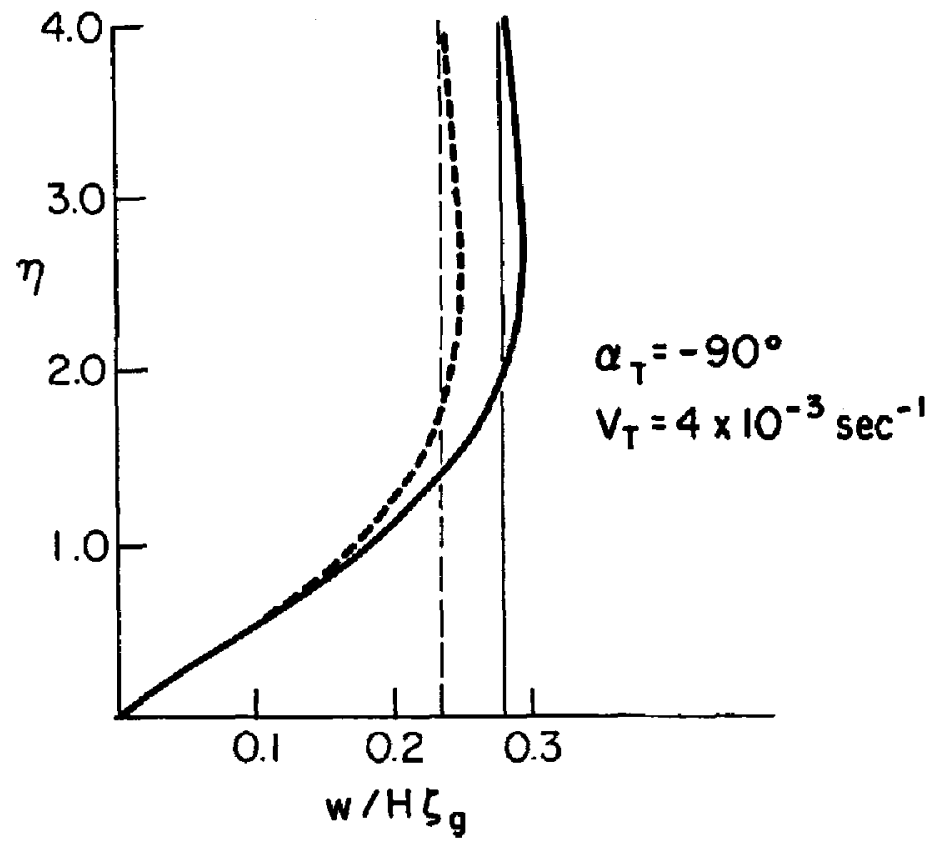

Fig. 12. The vertical velocity of the Ekman solution as a function of height computed from (6.7). Dashed line represents the barotropic solution.

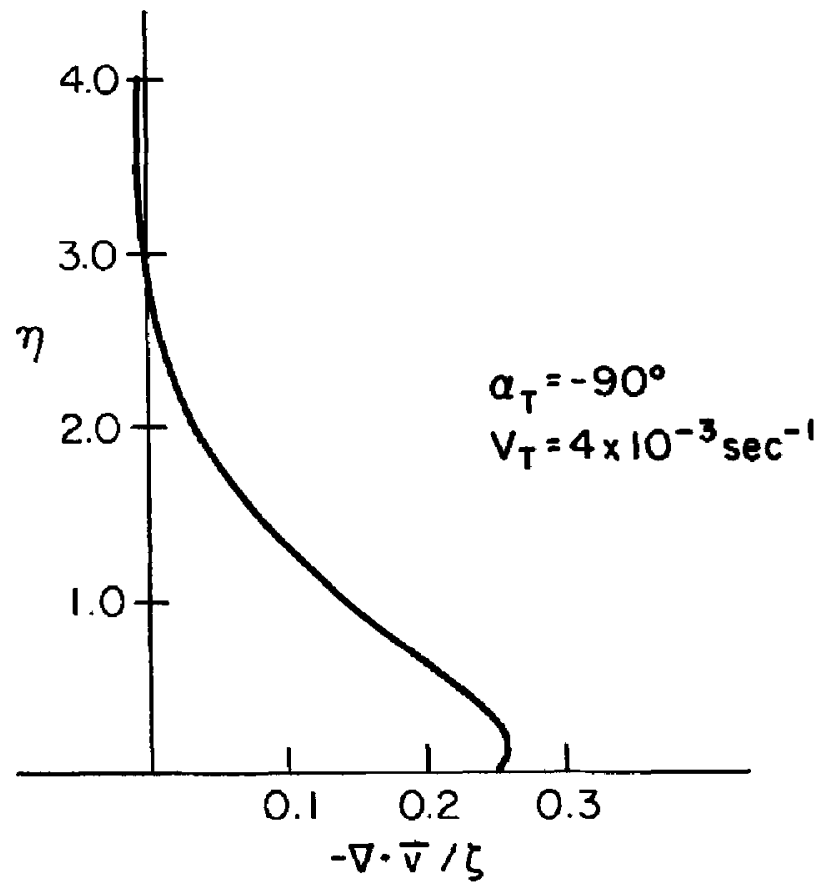

Fig. 13. The ratio of convergence and vorticity of the Ekman solution as a function of height. 
Figure 15 gives the ratio $\zeta / \zeta_{g 0}$ at $\eta=0$ as a function of $\alpha_{T}$ (solid curve). There is a very small variation in this case, and the typical value is about 0.6 . On the other hand, if we disregard the influence of the thermal wind in (5.5), we get the dashed curve in Figure 15 which shows variations from a maximum value of more than 0.7 to a minimum value of about 0.5 .

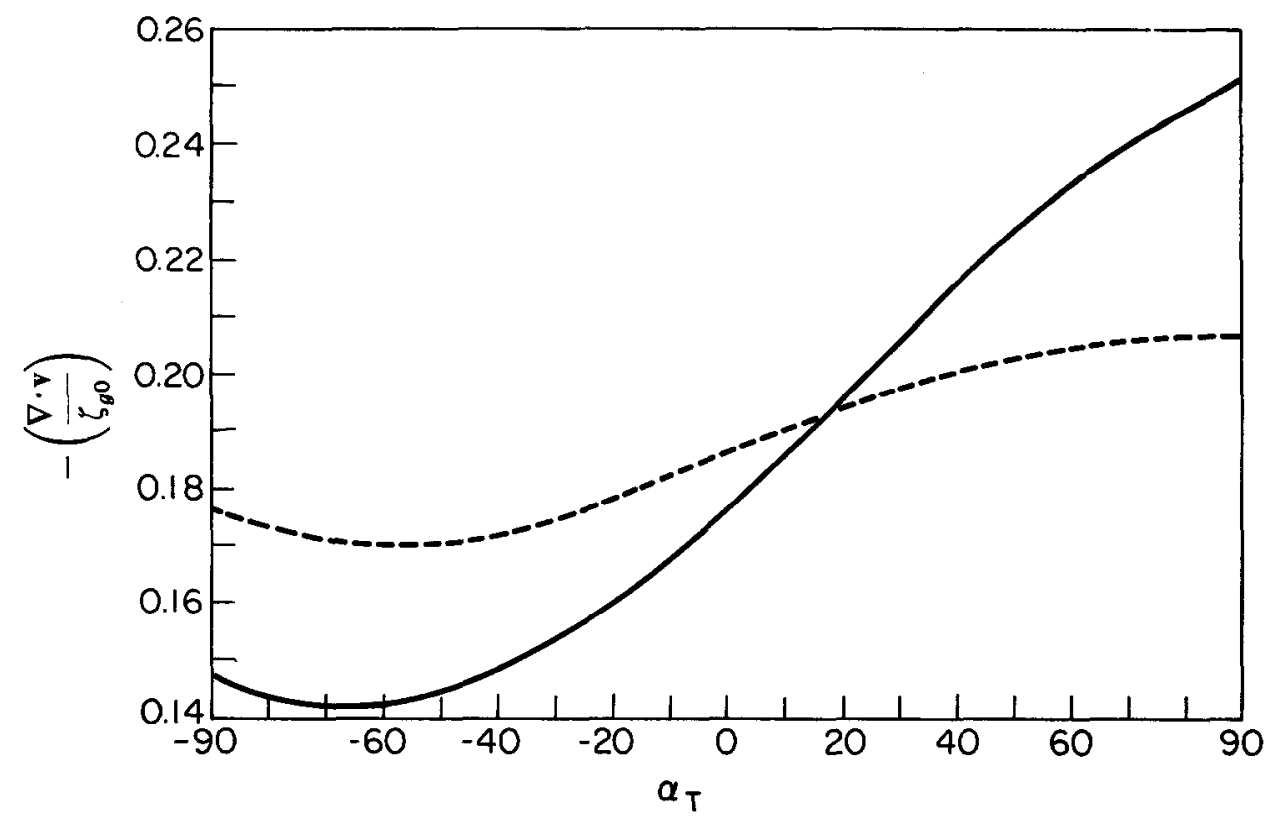

Fig. 14. The ratio of convergence and vorticity at $\eta=0$ as a function of $\alpha_{T}$. Dashed line represents the barotropic solution.

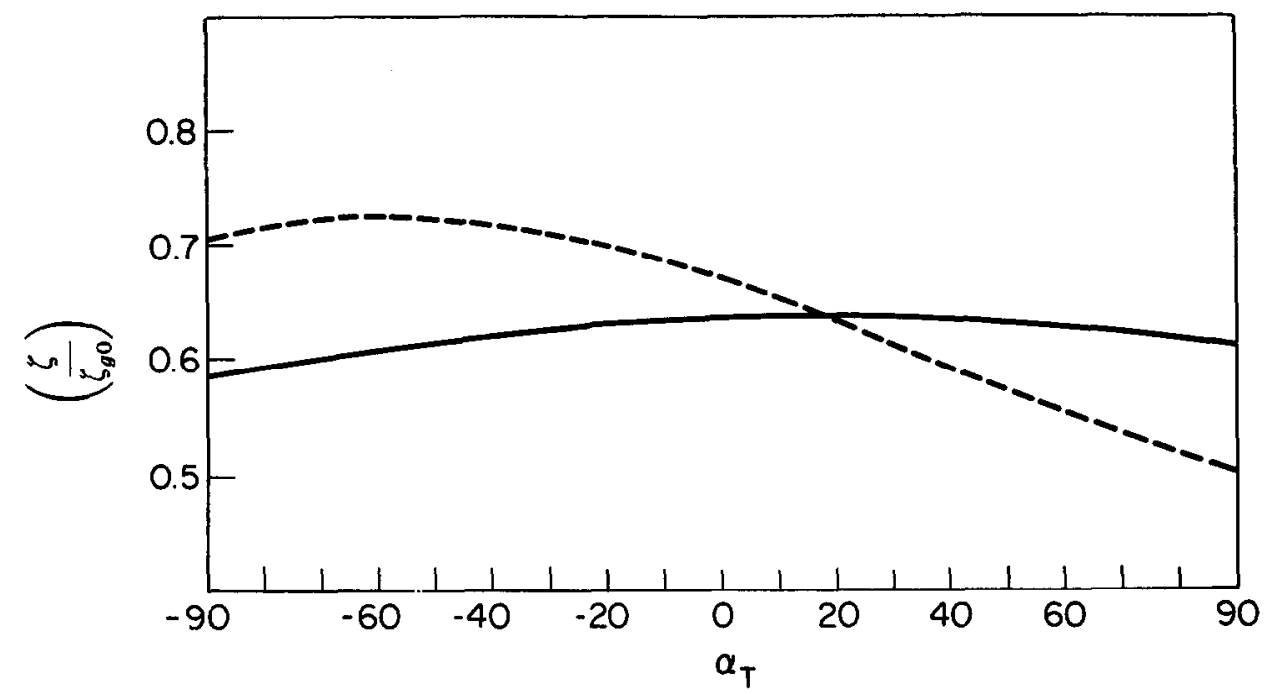

Fig. 15. The ratio of the vorticity and the geostrophic vorticity at $\eta=0$ as a function of $\alpha_{T}$. Dashed line is the barotropic solution. 


\section{Concluding Remarks}

The present study is an extention of the classical Ekman-Taylor treatment of the planetary boundary layer to include the effects of a thermal wind. In addition, we have used the continuity of the stress across the interface between the Prandtl and Ekman layers to determine the angle between the wind and the geostrophic wind at the interface as a function of the speed and direction of the thermal wind and the other parameters in the problem. Using a couple of simplifying assumptions regarding the variations in the horizontal directions of $V_{T}$ and $\alpha_{T}$, we have furthermore given the vertical variations of the horizontal divergence, the vorticity and the vertical velocity within the Ekman layer. It should be pointed out that if these simplifying assumptions are dropped, it is no longer possible to express the quantities mentioned above in terms of the geostrophic and thermal vorticities, and other combinations of the horizontal derivatives of the wind components will enter the picture.

Apart from the extensions made above, we have not attempted to generalize the standard treatment of the Ekman layer. The restrictive assumptions concerning the balance of forces under vanishing acceleration and the neglect of the variations of the eddy diffusivity have thus been maintained.

In spite of the limiting assumptions, it is nevertheless indicated by this study that there are important effects caused by the thermal wind which have a marked influence on some of the properties of the Ekman layer. There are several natural extensions of the present investigation. Foremost among these is the incorporation of a vertical variation of $K$, which perhaps can be accomplished using the methods of either Lettau and Dabberdt (1970) or Blackadar (1962). In any case, it will be important to study the role played by baroclinicity in the planetary boundary layer in a more realistic model as emphasized by Deardorff (1972) based on the possibility that the frictional layer may extend throughout the troposphere, as pointed out by Sheppard (1958).

\section{Acknowledgements}

This study was supported by the National Science Foundation under Grant GA16.166. The author furthermore wants to thank Mr James Pfaendtner who was in charge of all computer programming in connection with this study.

\section{References}

Blackadar, A. K.: 1962, 'The Vertical Distribution of Wind and Turbulent Exchange in a Neutral Atmosphere', J. Geophys. Res. 67, 3095-3102.

Charney, J. and Eliassen, A.: 1949, 'A Numerical Method for Predicting the Perturbations of the Middle Latitude Westerlies', Tellus 1, 38-54.

Deardorff, J. W.: 1972, 'Parameterization of the Planetary Boundary Layer for Use in General Circulation Models', Mon. Weather Rev. 100, 93106.

Godske, K. C. et al.: 1957, Dynamic Meteorology and Weather Forecasting, Amer. Meteorol. Soc., Boston, $800 \mathrm{pp}$.

Haltiner, G. J. and Martin, F. L.: 1957, Dynamical and Physical Meteorology, McGraw-Hill Book Co., New York, 470 pp. 
Lettau, H. H. and Dabberdt, W. F.: 1970, 'Variangular Wind Spirals', Boundary-Layer Meteorol. 1, 64-79.

MacKay K. P.: 1971 'Steady State Hodographs in a Baroclinic Boundary Layer', Boundary-Layer Meteorol. 2, 161-168.

Mahrt, L. J. and Schwerdtfeger, W.: 1970, 'Ekman Spirals for Exponential Thermal Wind', BoundaryLayer Meteorol. 1, 137-145.

Mendenhall, B. R.: 1967, 'A Statistical Study of the Frictional Wind Veering in the Planetary Boundary Layer', Atmos. Sci. Paper No. 116, Colorado State University, Fort Collins, 57 pp.

Petterssen, S.: 1956, Weather Analysis and Forecasting, 1, McGraw-Hill Book Co., New York, $428 \mathrm{pp}$.

Sheppard, P. A.: 1958, 'Transfer Across the Earth's Surface and Through the Air Above', Quart. J. Roy. Meteorol. Soc. 84, 205-229.

Taylor, G. I.: 1915, 'Eddy Motion in the Atmosphere', Phil. Trans. Roy. Soc. London A, 215, 1-26.

Taylor, G. I.: 1916, 'Skin Friction of the Wind on the Earth's Surface', Proc. Roy. Soc. London A, 92, 196-199. 